\title{
On the Lagrangian description of steady surface gravity waves
}

\author{
DIDIER CLAMOND \\ Institutt For Energiteknikk, P O Box 40, 2027 Kjeller, Norway \\ didier.clamond@ife.no
}

(Received 14 September 2006 and in revised form 10 June 2007)

This paper concerns the mathematical formulation of two-dimensional steady surface gravity waves in a Lagrangian description of motion. It is demonstrated first that classical second-order Lagrangian Stokes-like approximations do not exactly represent a steady wave motion in the presence of net mass transport (Stokes drift). A general mathematically correct formulation is then derived. This derivation leads naturally to a Lagrangian Stokes-like perturbation scheme that is uniformly valid for all time - in other words, without secular terms. This scheme is illustrated, both for irrotational waves, with seventh-order and third-order approximations in deep water and finite depth, respectively, and for rotational waves with a third-order approximation of the Gerstner-like wave on finite depth. It is also shown that the Lagrangian approximations are more accurate than their Eulerian counterparts of the same order.

\section{Introduction}

Although numerical models for surface gravity waves are undergoing constant improvement - in terms of speed, accuracy and generality (Fenton 1999; Dias \& Bridges 2006) - simplified analytical models remain (and will remain) in common use. Indeed, such models provide insight and are also accurate enough for many practical applications. The simplest analytical models are the linear ones, but there are many phenomena that cannot be described properly by linear solutions. For example, problems involving steep waves often require high-order approximations. Because the Lagrangian description of motion is intrinsically appropriate for describing steep waves, it is natural to use this description for deriving simple analytical models. Nevertheless, the vast majority of analytical investigations are performed using either the Eulerian description of motion or conformal mapping for two-dimensional irrotational flows, though the number of papers using a Lagrangian description have increased in recent years.

In the linear approximation, Lagrangian and Eulerian descriptions have similar mathematical complexity. This similarity does not occur at higher orders where algebraic manipulations in Lagrangian form are tedious. This is one reason why the Eulerian approach is generally preferred. In the pre-computer age, when calculations had to be performed by hand, it made sense to choose the method yielding the simpler derivations. This argument is no longer valid since complex algebraic manipulations can be achieved in seconds using a computer algebra system on a desktop computer. Hence, today, the most appropriate formulation should be used, regardless of the algebraic complexity of the derivation. The ability to manipulate algebra is not all, 
however, and one must also ensure that the assumed mathematical form of the solution can represent the phenomenon under investigation. This assumption is not always obvious, however. This point is illustrated here with steady waves in the Lagrangian description. Among all waves, steady solutions are of most importance because complex sea states are often analysed in terms of superpositions and interactions of these waves. Such an interpretation requires, in the first place, that steady solutions are properly described.

Using the velocity potential and the streamfunction as independent variables (which conformably map the fluid domain onto a half-plane), Stokes (1880) derived a fifthorder approximation for irrotational waves in deep water (see also Craik 2005). Several high-order approximations have been subsequently obtained following Stokes' approach (e.g. Wilton 1914). This type of approximation is now referred to as a Stokes expansion or Stokes wave. A similar expansion has also been used to investigate rotational waves (Gouyon 1958). Schwartz (1974) was the first to use a computer to compute explicitly high-order Stokes approximations. Like Stokes, he used conformal mapping to simplify the algebra. However, Drennan, Hui \& Tenti (1992) have shown that the Stokes expansion using conformal mapping (here in after named the Stokesian approximation) has a much smaller radius of convergence than its Eulerian counterpart (i.e. working in the physical plane).

There are comparatively few attempts to use similar techniques in the Lagrangian description of motion. Stokes (1847) derived a second-order Lagrangian approximation for irrotational waves. He found that the particle trajectories are not closed, thus leading to a net mass transport in the direction of the wave propagation; this nonlinear phenomenon is now called Stokes drift. Stokes' second-order approximation was subsequently extended to rotational waves (Miche 1944). Buldakov, Taylor \& Eatock-Taylor (2006) computed higher-order irrotational approximations according to Stokes' perturbation scheme. They noted that, starting from the third order, the solution is not bounded; the wave amplitude grows indefinitely in time. This is clearly due to unphysical secular terms. To overcome this problem, Buldakov et al. (2006) proposed a modified perturbation scheme where the particles are recursively relabelled at each order. This is an interesting, but rather involved, approach. However, Buldakov et al. (2006) do not explain the reason for the appearance of these secular terms. This is one of the purposes of the present paper where it is shown that these secular terms appear owing to a mathematical misrepresentation of steady waves. Abrashkin \& Zen'kovich (1990) and Chang, Liou \& Su (2007) avoided secular terms in their approximations by allowing the phase velocity to be a function of the 'vertical' Lagrangian coordinate. Chang et al. (2007) justified this technique with some heuristic considerations. It is demonstrated here that uniformly valid approximations of steady waves can indeed be obtained this way, but this is not the only possibility.

The paper is organized as follows. In $\S 2$, we briefly introduce the well-known Eulerian and Lagrangian equations of motion for steady two-dimensional gravity waves propagating at the impermeable surface of an incompressible perfect fluid. In $\S 3$, it is demonstrated that the classical second-order Lagrangian approximations of gravity waves do not represent exactly steady motions with mass transport, i.e. steadiness is not satisfied identically, but only up to the second order. As a consequence, secular terms appear at higher orders and these approximations are thus not uniformly valid for all times. This raises the problem of defining a steady motion using the Lagrangian description. This is solved in $\S 4$ for two-dimensional flows of incompressible fluids, where the general Lagrangian mathematical form of 
steady waves is derived, as well as some simplified forms that are convenient for practical applications. In $\S 5$, a uniformly valid Stokes-like perturbation scheme is introduced. It allows the construction of approximations without secular terms, and is hence valid for all times. This is illustrated: (i) for deep-water irrotational waves with a seventh-order approximation in $\S 6$; (ii) for finite-depth irrotational waves with a third-order approximation in $\S 7$; (iii) for finite-depth rotational waves without net mass transport with a third-order approximation in $\S 8$.

\section{Definitions, hypothesis and equations}

We consider steady two-dimensional gravity waves propagating at the surface of an incompressible fluid of constant density. The surface tension is neglected, $g$ denotes the acceleration due to gravity and the pressure is zero at the impermeable free surface. The fluid mean depth $d$ is either infinite or finite with a fixed horizontal impermeable sea bed.

\subsection{Eulerian description}

Let $(x, y)$ be, respectively, the horizontal coordinate and the upward vertical coordinate in the frame of reference moving with the wave (where the flow is steady), while $t$ denotes the time. The wave is $(2 \pi / k)$-periodic in the $x$-direction, with its crest at $x=0 . y=-d$ and $y=\eta(x)$ denote, respectively, the positions of the impermeable horizontal sea bed and the free surface, while $y=0$ is the (Eulerian) mean water level implying that

$$
\int_{-\pi / k}^{\pi / k} \eta(x) \mathrm{d} x=0 .
$$

$u \equiv \mathrm{D} x / \mathrm{D} t$ and $v \equiv \mathrm{D} y / \mathrm{D} t$ denote the horizontal and vertical velocities, respectively, $\mathrm{D} / \mathrm{D} t$ being the temporal derivative following the motion. The mean flow is

$$
\frac{k}{2 \pi} \int_{-\pi / k}^{\pi / k} \int_{-d}^{\eta} u \mathrm{~d} y \mathrm{~d} x \equiv-c d,
$$

$c$ being thus the wave phase velocity observed in the frame of reference without mean flow (but possibly with net transport at a given depth), which is positive if the wave propagates in the direction of increasing $x$. Note that $c$ is not the linear phase velocity and it is the sole definition (2.2) that is used everywhere in this paper.

The condition of incompressibility is fulfilled by introducing a streamfunction $\psi(x, y)$ defined with $u=\psi_{y}, v=-\psi_{x}$ and such that $\psi=0$ at the free surface (hence $\psi=d c$ at the bottom). We note in passing that $\mathrm{D} \psi / \mathrm{D} t=0$, so $\psi$ is independent of time in the Lagrangian description of motion.

The conservation of momentum implies, first, that the vorticity $\omega \equiv v_{x}-u_{y}$ is a function of $\psi$ only $(\omega=\omega(\psi))$ and, secondly, the Bernoulli equation

$$
2 P+2 g y+u^{2}+v^{2}=B(\psi),
$$

where $B$ is the Bernoulli 'constant' and $P$ is the pressure divided by the density.

For irrotational motions, $B$ is independent of $\psi$ and $B=c^{2}$ in deep water $(d \rightarrow \infty)$ and for the solitary wave $(k \rightarrow 0)$. It is also convenient to introduce a velocity potential $\phi$ such that $u=\phi_{x}$ and $v=\phi_{y}$ and so the condition $\omega=0$ is fulfilled identically.

\subsection{Lagrangian description}

In the Lagrangian description of motion, the (now dependent) variables $x$ and $y$ denote the position of a particle at time $t$ and are functions of $\left(x_{0}, y_{0}\right)$, the particle 
position at the initial time $t=0$. The velocities are related to the positions as

$$
u=\mathrm{D} x / \mathrm{D} t=x_{t}\left(x_{0}, y_{0}, t\right), \quad v=\mathrm{D} y / \mathrm{D} t=y_{t}\left(x_{0}, y_{0}, t\right),
$$

and the incompressibility equation and vorticity definition are

$$
\frac{\partial(x, y)}{\partial\left(x_{0}, y_{0}\right)}=1, \quad \omega=\frac{\partial\left(x_{t}, x\right)}{\partial\left(x_{0}, y_{0}\right)}+\frac{\partial\left(y_{t}, y\right)}{\partial\left(x_{0}, y_{0}\right)},
$$

whereas, for steady flows, the conservation of momentum can be reduced to the Bernoulli equation (2.3). Various relations and detailed derivations in the Lagrangian description are given by Lamb (1932) and Wehausen \& Laitone (1960), among others.

Using the initial positions $\left(x_{0}, y_{0}\right)$ as independent variables is not convenient because the initial fluid domain is generally complicated and unknown. Moreover, a travelling wave of permanent form cannot be described simply by functions of $x_{0}-c t$. Indeed, if it were the case, the horizontal position, for example, would be $x=x\left(x_{0}-c t, y_{0}\right)$ and hence at the surface $x=x\left(x_{0}-c t, \eta\left(x_{0}\right)\right)$ which is a function of $x_{0}-c t$ only if $\eta\left(x_{0}\right)=0$, i.e. for a flat surface.

Thus, it is easier to solve the equations using the coordinate transformation $\left(x_{0}, y_{0}\right) \mapsto(\alpha, \beta)$ that maps the physical fluid domain at $t=0$ onto the strip $-d \leqslant \beta \leqslant 0$; $\beta=0$ and $\beta=-d$ corresponding to the free surface $y=\eta$ and to the sea bed $y=-d$, respectively. This transformation (particle relabelling) and its inverse are considered univalued, continuous and differentiable. Hence, the Jacobian $J$ of the transformation satisfies

$$
J(\alpha, \beta) \equiv \frac{\partial\left(x_{0}, y_{0}\right)}{\partial(\alpha, \beta)}>0,
$$

and the incompressibility equation and vorticity definition become, respectively,

$$
\frac{\partial(x, y)}{\partial(\alpha, \beta)}=J, \quad \frac{\partial\left(x_{t}, x\right)}{\partial(\alpha, \beta)}+\frac{\partial\left(y_{t}, y\right)}{\partial(\alpha, \beta)}=J \omega .
$$

There are, of course, an infinite number of such transformations.

The definition of the steady streamfunction $\psi(x, y)$ yields

$$
\mathrm{d} \psi=u \mathrm{~d} y-v \mathrm{~d} x=\left(u y_{\alpha}-v x_{\alpha}\right) \mathrm{d} \alpha+\left(u y_{\beta}-v x_{\beta}\right) \mathrm{d} \beta+\left(u y_{t}-v x_{t}\right) \mathrm{d} t,
$$

thence $\psi=\psi(\alpha, \beta)$ with

$$
\psi_{\alpha}=x_{t} y_{\alpha}-y_{t} x_{\alpha}=\frac{\partial(x, y)}{\partial(t, \alpha)}, \quad \psi_{\beta}=x_{t} y_{\beta}-y_{t} x_{\beta}=\frac{\partial(x, y)}{\partial(t, \beta)} .
$$

Equations (2.9) define explicitly the streamfunction in the Lagrangian description.

\subsection{Remarks}

In this paper, the Eulerian averaging $(k / 2 \pi) \int_{-\pi / k}^{\pi / k} \bullet \mathrm{d} x$ is used to define all mean quantities (even when using the Lagrangian description of motion) because it is the most common and convenient, but another averaging can also be used (LonguetHiggins 1986).

The speed $c$, as defined by (2.2), is sometimes called the Stokes phase velocity. It is also customary (e.g. Williams 1981) to consider the so-called Euler phase velocity $c^{\prime}$ observed in the frame of reference without mean velocity at the bottom, i.e.

$$
-c^{\prime} \equiv \frac{k}{2 \pi} \int_{-\pi / k}^{\pi / k} u(x, y=-d) \mathrm{d} x .
$$

$c^{\prime} \neq c$ in general, but for irrotational flows, $c^{\prime}=c$ in deep water and for solitary waves. 


\section{The problem of Lagrangian steady waves}

Stokes $(1847, \S 9)$ derived a second-order Lagrangian approximation for irrotational waves in deep water which, in the present notations, is

$$
k x=k \xi+\varepsilon^{2} c t \mathrm{e}^{2 k \beta}-\varepsilon \mathrm{e}^{k \beta} \sin k \xi, \quad k y=k \beta+\frac{1}{2} \varepsilon^{2}+\varepsilon \mathrm{e}^{k \beta} \cos k \xi,
$$

where $\xi=\alpha-c t, k$ is the wavenumber and $\varepsilon$ is half the total wave height multiplied by $k$ (steepness). Note that Stokes' original approximation does not incorporate the term $\varepsilon^{2} / 2$ in $(3.1 b)$; it is introduced here to ensure that $\eta=y(\xi, 0)$ averages to zero according to (2.1). Stokes (1847) derived also a second-order approximation on finite depth-but he did not give the full explicit solution; see Wehausen \& Laitone (1960, equation (27.41)) - and Miche (1944) extended these approximations to rotational flows. All these second-order approximations can be formally written as

$$
x=\xi+\gamma(\beta) t+X(\xi, \beta), \quad y=\beta+Y(\xi, \beta),
$$

where $X$ and $Y$ are bounded periodic functions (Fourier polynomials) of $\xi=\alpha-c t$ and $\gamma$ characterizes the Stokes drift. On finite depth $Y=0$ at the bottom $\beta=-d$, while in deep water the wave has no influence far below the surface so $\{\gamma ; X ; Y\} \rightarrow 0$ as $\beta \rightarrow-\infty$. If $\gamma(\beta)=0$ there is no Stokes drift because the particle horizontal coordinate $x_{F}$ observed in the frame of reference without mean flow (where the wave appears to travel with the speed $c$ ), i.e.

$$
x_{F} \equiv x+c t=\alpha+\gamma(\beta) t+X(\alpha-c t, \beta),
$$

is a periodic function in time when $\gamma=0$. Note that Gerstner's exact rotational solution (cf. Appendix A) is of the form (3.2), but without Stokes' drift $(\gamma=0)$. Note also that we could consider a generalization of (3.2) such that $\gamma=\gamma(\alpha, \beta)$, but we shall see that this is of no interest here, however.

We can also consider higher-order approximations. Using Stokes' classical perturbation scheme, the third- and higher-order approximations involve secular terms; the wave amplitude increases indefinitely in time (Buldakov et al. 2006) and these approximations are therefore not uniformly valid for all times (though they are mathematically correct from an asymptotic viewpoint). Thus, for practical applications, these high-order approximations must be recast (via, e.g. Lie group transformations, renormalization, etc.) into forms that are uniformly valid for all times. In order to apply such transformations, it is necessary to know the form of solution that the approximations must be transformed to. It is natural to try first to recast the approximations into the form (3.2). However, this cannot be achieved.

THEOREM 1. No exact solution describing a steady wave with Stokes' drift can be obtained in the form (3.2).

Proof. The substitution of the form of solution (3.2) with $\gamma=\gamma(\alpha, \beta)$ into equations (2.9) yields

$$
\psi_{\alpha}=\left(\gamma+c \gamma_{\alpha} t\right) y_{\xi}, \quad \psi_{\beta}=\gamma y_{\beta}+c \gamma_{\beta} t y_{\xi}+\frac{\partial(\xi+X, y)}{\partial(t, \beta)} .
$$

In these relations, all the terms are bounded periodic functions of time except the explicit terms $t$. Therefore, the relations can be fulfilled for all times only if the coefficients of $t$ vanish, implying that $\gamma$ is a constant and that there is thus no Stokes' drift. Further, the free surface being a streamline, $\psi_{\alpha}=\gamma y_{\xi}=0$ at $\beta=0$ and hence $\gamma=0$. (In this discussion the trivial cases $c=0$ and $y_{\xi}(\xi, 0)=0$ were disregarded.) 
In conclusion, no uniformly valid steady solutions can be obtained in the form (3.2) when the Stokes drift is present.

Then the question arises: What is the general mathematical form of a steady wave in the Lagrangian description of motion? As just shown, this question is not as trivial as it may sound at first. The answer is necessary for the construction of high-order approximations (and exact solutions) for steady wave motions that are uniformly valid for all times.

\section{Lagrangian description of a steady wave motion}

By definition, a steady wave motion is such that the flow appears independent of time at all fixed positions $(x, y)$. (A travelling wave of permanent form is such that there exists a frame of reference where, via a Galilean transformation, the flow appears steady.) As stated, steadiness is essentially an Eulerian concept and therefore its Lagrangian counterpart must be made precise. Obviously, a steady flow in the Lagrangian description cannot be defined as independent of time because that would imply the absence of motion. This section is devoted to the derivation of the general Lagrangian formulation of steady flows, as well as some simplified formulations.

\subsection{General coordinates}

In the Eulerian description of motion, if the streamfunction is independent of time, it implies (via the $\psi$ definition) that the velocity is also constant in time. Therefore, the sole condition $\psi_{t}=0$ is sufficient to define completely a steady flow. In the Lagrangian description of steady flows, the particle positions are varying in time, as already mentioned, but we have seen that the streamfunction is independent of time, i.e. $\psi=\psi(\alpha, \beta)$. This latter feature is sufficient to define explicitly a steady flow, as in the Eulerian case.

The Eulerian definition of $\psi$ is transformed into the Lagrangian form as

$$
\begin{gathered}
u=\frac{\partial \psi}{\partial y}=\frac{\partial(x, \psi)}{\partial(x, y)} \Longleftrightarrow \frac{\partial x}{\partial t}=\frac{1}{J} \frac{\partial(x, \psi)}{\partial(\alpha, \beta)}, \\
v=-\frac{\partial \psi}{\partial x}=\frac{\partial(y, \psi)}{\partial(x, y)} \Longleftrightarrow \frac{\partial y}{\partial t}=\frac{1}{J} \frac{\partial(y, \psi)}{\partial(\alpha, \beta)} .
\end{gathered}
$$

Then, because $\psi$ is independent of $t,(4.1 b-d)$ are easily solved taking $(\alpha, \psi, t)-$ or $(\beta, \psi, t)-$ as independent variables and, after integration, returning to the original variables $(\alpha, \beta, t)$. Thus, we obtain easily

$$
x=\Xi(\tau, \psi), \quad y=\Upsilon(\tau, \psi), \quad \tau \equiv t+T(\alpha, \beta), \quad \frac{\partial(T, \psi)}{\partial(\alpha, \beta)}=J,
$$

$\Xi$ and $\Upsilon$ being unspecified functions. The solution (4.2) is the most general Lagrangian formulation of steady two-dimensional flows for incompressible fluids. Note that, with (4.2), the mass conservation (2.7a) and the two relations (2.9) yield the single equation

$$
\frac{\partial(\Xi, \Upsilon)}{\partial(\tau, \psi)}=1
$$

In summary, a steady wave motion is expressed in terms of functions of $t+T(\alpha, \beta)$ - with $T$ given by (4.2d) - and not in terms of functions of $t-\alpha / c$, in general. The latter possibility can be obtained with some changes of variables, however, as shown below. Indeed, $T$ is defined implicitly by the auxiliary equation $(4.2 d)$ that has 
infinitely many solutions; this reflects the fact that the particles can be labelled in infinitely many ways. It is thus of practical interest to introduce special coordinates such that the solution is simpler.

\subsection{Special coordinates such that $\psi=\psi(\beta)$}

Since the free surface and the bottom are streamlines, and because the streamfunction is independent of time, it is natural for the sake of simplicity to chose parametric variables (particle labels) such that each streamline is also an iso- $\beta$ line, i.e. $\psi=\psi(\beta)$. With this particular choice of variables, the solution (4.2) becomes

$$
x=\Xi(\tau, \beta), \quad y=\Upsilon(\tau, \beta), \quad \tau=t+\left(\frac{\mathrm{d} \psi}{\mathrm{d} \beta}\right)^{-1} \int J \mathrm{~d} \alpha .
$$

This simplified solution is still too general for most practical applications and further simplifications are desirable.

\subsection{Simplified coordinates such that $\psi=\psi(\beta)$ and $J=J(\beta)$}

The solution would obviously be simpler if $\psi=\psi(\beta)$ together with $J=J(\beta)$, a situation that can be obtained by relabelling the particles as follows.

Starting from the simplified coordinates such that $\psi=\psi(\beta)$, the change of coordinates (particle relabelling)

$$
\alpha^{\star}(\alpha, \beta) \equiv-\int \frac{C J}{\psi_{\beta}} \mathrm{d} \alpha, \quad \beta^{\star}(\beta) \equiv-\int \frac{j \psi_{\beta}}{C} \mathrm{~d} \beta,
$$

where $C$ and $j$ are arbitrary regular functions of $\beta$, yields

$$
\frac{\partial\left(\alpha^{\star}, \beta^{\star}\right)}{\partial(\alpha, \beta)}=j(\beta) J(\alpha, \beta), \quad \tau=t-\frac{\alpha^{\star}}{C(\beta)} .
$$

The mass conservation $(2.7 a)$ then becomes

$$
\frac{\partial(x, y)}{\partial\left(\alpha^{\star}, \beta^{\star}\right)}=\frac{1}{j(\beta)} \equiv J^{\star}\left(\beta^{\star}\right),
$$

showing that it is possible to use coordinates such that both $\psi$ and $J$ depend only on the particle 'vertical' label.

Thus, with the simplified variables $\left(\alpha^{\star}, \beta^{\star}\right)$ and introducing $\xi \equiv-C \tau=\alpha^{\star}-C t-$ but hereinafter omitting the stars for brevity - the equations of incompressibility, vorticity and Bernoulli become, respectively,

$$
\begin{aligned}
J=\frac{\partial(x, y)}{\partial(\xi, \beta)} & =-\frac{1}{C} \frac{\mathrm{d} \psi}{\mathrm{d} \beta}, \\
\frac{\partial\left(x_{\xi}, x\right)}{\partial(\xi, \beta)}+\frac{\partial\left(y_{\xi}, y\right)}{\partial(\xi, \beta)}-\frac{x_{\xi}^{2}+y_{\xi}^{2}}{C} \frac{\mathrm{d} C}{\mathrm{~d} \beta} & =-\frac{J \omega}{C}, \\
2 P+2 g y+\left(C x_{\xi}\right)^{2}+\left(C y_{\xi}\right)^{2} & =B,
\end{aligned}
$$

where $\psi, B, C$ and $J$ are functions of $\beta$ only; while $P, x$ and $y$ are functions of both $\xi$ and $\beta$. Note that $\xi$ is not a Lagrangian variable because it depends on $t$. This modified Lagrangian variable was apparently first introduced by Abrashkin \& Zen'kovich (1990) who showed that it can be conveniently used to describe waves with mass transport; it is also used by Chang et al. (2007) who give a fifth-order irrotational approximation on finite depth. 


\subsection{Normalized coordinates}

We have seen that $x=x(\xi, \beta)$ and $y=y(\xi, \beta)$, with $\xi=\alpha-C(\beta) t$, is a general form of steady waves under the constraints $J=J(\beta)$ and $\psi=\psi(\beta)$. Further simplifications are actually possible. For instance, via particle relabelling, we can take $C=$ constant $=c$ together with $J=1$, without loss of generality. This can be easily seen taking $C(\beta)=c$ and $j(\beta)=1$ in (4.5), which is a possible choice because these variables are arbitrary. It can also be seen directly from the pseudo-Lagrangian $\xi$-variable when rewritten as

$$
\xi=\alpha-C t=(C / c)[(c / C) \alpha-c t]=(C / c)\left[\alpha^{\dagger}-c t\right],
$$

where $\alpha^{\dagger} \equiv \alpha c / C(\beta)$ and introducing $\beta^{\dagger} \equiv \int_{0}^{\beta}(C / c) J \mathrm{~d} \beta$. Note that in (4.9), the definition (2.2) of $c$ is not used, meaning that (via particle relabelling) a steady wave motion can always be expressed as a function of $\alpha-c t$, independently of the way $c$ is defined.

Lagrangian variables such that $J=1$ are sometimes called Miche's coordinates (named after Miche 1944). Dealing with Miche's variables is not always a good idea, however, because such a choice restricts the possible simple forms of solution and complicates the derivations. For instance, the well-known Gerstner exact rotational wave has a very simple algebraic expression with $J \neq 1$. It is algebraically more complicated, and practically not advantageous, to rewrite Gerstner's solution so that $J=1$ (see note (iii) in Appendix A). Other illustrations of this point are demonstrated below for irrotational and Gerstner-like waves on fluid of finite depth.

\subsection{Remarks}

A conclusion of this section is that the mathematical form of a steady flow is not independent of the choice of the particle labels. Conversely, if a special form of solution is introduced a priori, we must check that there indeed exists a special coordinate system leading to this peculiar form. This is not always the case, as shown above with the expression (3.2). This claim remains a fortiori true for unsteady flows for which the correct mathematical expression of a given flow will be more difficult to derive, in general.

The general form of solution (4.2) derives from kinematical considerations only. It is therefore also valid for heterogeneous viscous fluids over a non-horizontal sea bed and in the presence of surface tension, provided that the fluid is incompressible and in steady two-dimensional motion. The generalizations in three dimensions and for compressible fluids are given in the Appendix C.

\section{Stokes-like perturbation scheme}

Having defined above the mathematical form of steady flows in the Lagrangian description of motion, it is now easy to introduce a perturbation scheme leading to approximate solutions that are uniformly valid for all times, for either rotational or irrotational flows, with or without mass transport.

\subsection{Fourier series}

In a frame of reference moving with the speed of the net mass transport along a given streamline (the corresponding particle trajectories appear closed), the wave has an apparent wavenumber $K$, an apparent velocity $C$ and an apparent period $2 \pi / K C$. The mass transport is (a priori) different for each streamline and therefore, owing to the Doppler effect, the parameters $K$ and $C$ are functions of $\psi$, in general. Hence, for $(2 \pi / k)$-periodic waves symmetric around the crest, it is natural to seek solutions that 
are expandable in Fourier series (using the simplified coordinates introduced in $\S 4.3$ ) as

$$
x=k^{-1} K \xi-\sum_{i=1}^{\infty} X_{i} \sin i K \xi, \quad y=\sum_{i=0}^{\infty} Y_{i} \cos i K \xi,
$$

with $\xi=\alpha-C t$ and where $K, C, X_{i}$ and $Y_{i}$ are functions of $\beta$ to be determined. Fourier series are uniformly convergent for continuous functions with a piecewise continuous first derivative, meaning that all periodic solutions of physical interest can be represented by the series (5.1). Other eigenfunctions could also be used, however.

Obviously, from $(5.1 a)$, the mass transport velocity is $-C K / k$ in the frame of reference moving with the wave, which is independent of $\beta$ in the absence of Stokes drift. Hence, the transport velocity is $c-C K / k$ in the frame of reference without mean flow.

Note that the expansion (5.1) is not of the form (3.2), even if $C$ is constant. However, substituting $c$ by $C=c+\gamma(\beta)$ into the functions $X$ and $Y$ of (3.2), the resulting expression takes the form (5.1) with $K=k$.

\subsection{Small-parameter expansion}

To solve the equations iteratively, the functions are further expanded in power series

$$
\left\{X_{i} ; Y_{i}\right\}=\sum_{j=0}^{\infty} \varepsilon^{i+j}\left\{X_{i, j} ; Y_{i, j}\right\}, \quad\{K ; C\}=\sum_{j=0}^{\infty} \varepsilon^{j}\left\{K_{j} ; C_{j}\right\},
$$

and the equations are fulfilled for all powers of $\varepsilon$ independently. The expansion parameter $\varepsilon$ is chosen here to be the wave steepness (half the total wave height multiplied by $k$ ), implying that

$$
\varepsilon=\sum_{i=1}^{\infty} \sum_{j=0}^{\infty} \varepsilon^{2 i+j-1} k Y_{2 i-1, j}(\beta=0)
$$

The relation (5.3) must also be satisfied for all powers of $\varepsilon$ independently, thus giving an extra set of equations necessary to close the problem.

Another expansion parameter could of course be used; the present expansion parameter being one of the most common and convenient, however. Some arguments for choosing $\varepsilon$ as the expansion parameter are given in the Appendix B. Owing to this particular choice, approximations and exact solutions will be compared with identical wavelength and steepness (in addition with identical parameters $g, d$, frame of reference, mean water level and position of the wave crest), but the solutions could also be compared with another pair of identical parameters (period, mean momentum flux, mean kinetic energy, etc.).

With this Stokes-like double series, we have enough free functions at our disposal to fulfil the equations at all orders. Actually, we have more freedom than required by the equations; extra relations are introduced imposing that the solution must be uniformly valid in the whole fluid and for all time, i.e. cancelling the secular terms.

When working with the non-normalized coordinates, there are still extra degrees of freedom and, to close the problem, we can impose two extra conditions among, e.g. $K=k, C=c, J=1, \psi=-\beta c$, or $Y_{0}$ being a linear function of $\beta$. However, not all of these constraints are independent and some cannot be imposed simultaneously. For instance, the constraints $K=k$ and $C=c$ cannot be used together for irrotational waves because that would imply the absence of Stokes' drift. 


\subsection{Remarks}

In his amplitude expansion of surface waves, Stokes expanded also the phase velocity in order to avoid secular terms in the solution. He could have instead expanded the wavenumber. The Stokes-like expansion in the Lagrangian description is similar with the additional feature that the wavenumber or phase velocity must be a function of $\psi$ in order to describe the net mass transport (if it is present).

Stokes-like expansions are not efficient in shallow water (i.e. for $k d \ll 1$ ) and an alternative perturbation scheme should hence be introduced. A Lagrangian shallowwater theory presents no particular difficulties, but it is out of the scope of the current work, where the motivation is to simply illustrate the advantage of the Lagrangian formulation.

\section{Application to irrotational waves in deep water}

The perturbation scheme above is applied to irrotational gravity waves $(\omega=0)$, considering only the low-order approximations of seventh-order for deep water. The goal here is simply to illustrate the efficiency of the Lagrangian formulation, and not to provide a thorough mathematical investigation.

\subsection{Seventh-order approximation}

In deep water, $B=c^{2}$. Far below the surface, the wave has no influence and therefore the solution must tend to a uniform current, i.e. $X_{i} \rightarrow 0, Y_{i>0} \rightarrow 0, K \rightarrow K_{\infty}$ and $C \rightarrow C_{\infty}$ as $\beta \rightarrow-\infty$. It is obvious that - via a change of definition of the $\alpha$-variable we can impose $K_{\infty}=k$ without loss of generality and thus $C_{\infty}=c$.

It is here advantageous (and possible) to impose $C=c$ and $\psi=-c \beta$, so that the solution is a function of $\alpha-c t$ and $\beta$ has a simple connection to the physical quantities $c$ and $\psi$; it also yields $J=1$. We are thus using the simplified normalized (Miche's) coordinates. To the seventh-order, after some algebra, we obtain

$$
\begin{aligned}
k Y_{0}= & k \beta-\frac{1}{2} \varepsilon^{2}+\frac{1}{2} \varepsilon^{4}+\frac{13}{24} \varepsilon^{6}+\left(\varepsilon^{2}-3 \varepsilon^{4}+\frac{13}{6} \varepsilon^{6}\right) \mathrm{e}^{2 k \beta} \\
& +\left(3 \varepsilon^{4}-15 \varepsilon^{6}\right) \mathrm{e}^{4 k \beta}+\frac{53}{4} \varepsilon^{6} \mathrm{e}^{6 k \beta}, \\
k X_{1}= & \left(\varepsilon-\frac{3}{2} \varepsilon^{3}-\frac{1}{24} \varepsilon^{5}-\frac{3007}{1440} \varepsilon^{7}\right) \mathrm{e}^{k \beta}+\left(\frac{5}{2} \varepsilon^{3}-\frac{35}{4} \varepsilon^{5}+\frac{475}{48} \varepsilon^{7}\right) \mathrm{e}^{3 k \beta} \\
& +\left(\frac{43}{4} \varepsilon^{5}-\frac{1369}{24} \varepsilon^{7}\right) \mathrm{e}^{5 k \beta}+\frac{441}{8} \varepsilon^{7} \mathrm{e}^{7 k \beta}, \\
k Y_{1}= & \left(\varepsilon-\frac{3}{2} \varepsilon^{3}-\frac{1}{24} \varepsilon^{5}-\frac{3007}{1440} \varepsilon^{7}\right) \mathrm{e}^{k \beta}+\left(\frac{3}{2} \varepsilon^{3}-\frac{21}{4} \varepsilon^{5}+\frac{95}{16} \varepsilon^{7}\right) \mathrm{e}^{3 k \beta} \\
& +\left(\frac{21}{4} \varepsilon^{5}-\frac{665}{24} \varepsilon^{7}\right) \mathrm{e}^{5 k \beta}+\frac{189}{8} \varepsilon^{7} \mathrm{e}^{7 k \beta}, \\
k X_{2}= & \left(\frac{1}{2} \varepsilon^{4}-\frac{7}{12} \varepsilon^{6}\right) \mathrm{e}^{2 k \beta}-\left(\frac{1}{6} \varepsilon^{4}-\frac{35}{12} \varepsilon^{6}\right) \mathrm{e}^{4 k \beta}-\frac{25}{18} \varepsilon^{6} \mathrm{e}^{6 k \beta}, \\
k Y_{2}= & \left(\frac{1}{2} \varepsilon^{4}-\frac{7}{12} \varepsilon^{6}\right) \mathrm{e}^{2 k \beta}-\left(\frac{1}{3} \varepsilon^{4}-\frac{10}{3} \varepsilon^{6}\right) \mathrm{e}^{4 k \beta}-\frac{22}{9} \varepsilon^{6} \mathrm{e}^{6 k \beta}, \\
k X_{3}= & \left(\frac{1}{12} \varepsilon^{5}+\frac{49}{144} \varepsilon^{7}\right) \mathrm{e}^{3 k \beta}-\left(\frac{1}{72} \varepsilon^{5}-\frac{85}{432} \varepsilon^{7}\right) \mathrm{e}^{5 k \beta}-\frac{31}{288} \varepsilon^{7} \mathrm{e}^{7 k \beta}, \\
k Y_{3}= & \left(\frac{1}{12} \varepsilon^{5}+\frac{49}{144} \varepsilon^{7}\right) \mathrm{e}^{3 k \beta}-\left(\frac{1}{24} \varepsilon^{5}-\frac{25}{144} \varepsilon^{7}\right) \mathrm{e}^{5 k \beta}-\frac{9}{32} \varepsilon^{7} \mathrm{e}^{7 k \beta}, \\
k X_{4}= & \frac{1}{72} \varepsilon^{6} \mathrm{e}^{4 k \beta}-\frac{1}{720} \varepsilon^{6} \mathrm{e}^{6 k \beta} \\
k Y_{4}= & \frac{1}{72} \varepsilon^{6} \mathrm{e}^{4 k \beta}-\frac{1}{180} \varepsilon^{6} \mathrm{e}^{6 k \beta}, \\
k X_{5}= & \frac{1}{480} \varepsilon^{7} \mathrm{e}^{5 k \beta}-\frac{1}{7200} \varepsilon^{7} \mathrm{e}^{7 k \beta}, \\
k Y_{5}= & \frac{1}{480} \varepsilon^{7} \mathrm{e}^{5 k \beta}-\frac{1}{1440} \varepsilon^{7} \mathrm{e}^{7 k \beta},
\end{aligned}
$$


and

$$
\begin{aligned}
K & =\left[1-\left(\varepsilon^{2}-3 \varepsilon^{4}+\frac{13}{6} \varepsilon^{6}\right) \mathrm{e}^{2 k \beta}-\left(3 \varepsilon^{4}-14 \varepsilon^{6}\right) \mathrm{e}^{4 k \beta}-\frac{53}{4} \varepsilon^{6} \mathrm{e}^{6 k \beta}\right] k, \\
c & =\left[1+\frac{1}{2} \varepsilon^{2}+\frac{1}{8} \varepsilon^{4}+\frac{1}{16} \varepsilon^{6}\right] \sqrt{g / k} .
\end{aligned}
$$

This Lagrangian Stokes-like approximation is free of secular terms and is therefore uniformly valid in the whole fluid domain and for all times; this remains true at higher orders. At the free surface $\beta=0$, this seventh-order Stokes-like approximation becomes

$$
\begin{aligned}
k x= & K \xi-\left(\varepsilon+\varepsilon^{3}+\frac{47}{24} \varepsilon^{5}+\frac{8483}{1440} \varepsilon^{7}\right) \sin K \xi-\left(\frac{1}{3} \varepsilon^{4}+\frac{17}{18} \varepsilon^{6}\right) \sin 2 K \xi \\
& -\left(\frac{5}{72} \varepsilon^{5}+\frac{371}{864} \varepsilon^{7}\right) \sin 3 K \xi-\frac{1}{80} \varepsilon^{6} \sin 4 K \xi-\frac{7}{3600} \varepsilon^{7} \sin 5 K \xi, \\
k \eta= & \frac{1}{2} \varepsilon^{2}+\frac{1}{2} \varepsilon^{4}+\frac{23}{24} \varepsilon^{6}+\left(\varepsilon-\frac{1}{24} \varepsilon^{5}-\frac{337}{1440} \varepsilon^{7}\right) \cos K \xi+\left(\frac{1}{6} \varepsilon^{4}+\frac{11}{36} \varepsilon^{6}\right) \cos 2 K \xi \\
& +\left(\frac{1}{24} \varepsilon^{5}+\frac{67}{288} \varepsilon^{7}\right) \cos 3 K \xi+\frac{1}{120} \varepsilon^{6} \cos 4 K \xi+\frac{1}{720} \varepsilon^{7} \cos 5 K \xi, \\
K= & {\left[1-\varepsilon^{2}-\frac{17}{12} \varepsilon^{6}\right] k . }
\end{aligned}
$$

Notice that the first harmonic (twice the fundamental frequency) appears at the fourth-order only, the second harmonic appears at the fifth-order, and so on. In Eulerian description too, the first harmonic appears only at the fourth-order for the velocity field (see (B 1a)), but it appears at the second-order for the surface elevation (see (B 1b)). Conversely, the first harmonic appears at second order in the Stokesian approximation (see (B 2)). At least for the low-order approximations, this shows the superiority of the Lagrangian formulation over the Eulerian and Stokesian formulations.

\subsection{Stokes drift}

The particle drift velocity $V$ observed in the frame of reference where the velocity of the fluid tends to zero as $y \rightarrow-\infty$ is

$$
V(\beta) \equiv c-k^{-1} K C,
$$

hence

$$
c^{-1} V=\left(\varepsilon^{2}-3 \varepsilon^{4}+\frac{13}{6} \varepsilon^{6}\right) \mathrm{e}^{2 k \beta}+\left(3 \varepsilon^{4}-14 \varepsilon^{6}\right) \mathrm{e}^{4 k \beta}+\frac{53}{4} \varepsilon^{6} \mathrm{e}^{6 k \beta}+O\left(\varepsilon^{8}\right) .
$$

At the surface, the drift velocity is therefore

$$
c^{-1} V(0)=\varepsilon^{2}+\frac{17}{12} \varepsilon^{6}+O\left(\varepsilon^{8}\right),
$$

a result first obtained by Longuet-Higgins (1987, equation 5.4d) via a different route. The particle trajectories are open orbits with a net mass transport in the direction of the wave propagation (figure $1(a)$ ). Subtracting the mass transport component, the resulting closed orbits are highly symmetric, even for steep waves (figure $1(b)$ ). Further details on these matters are given by Longuet-Higgins (1987).

\subsection{Comparison with other approximations and exact solution}

Comparisons of $(6.1)$ for a steep wave $(\varepsilon=0.424)$ with an exact numerical solution (Fenton 1988) and with seventh-order Eulerian and Stokesian approximations (Appendix B) show that it is accurate (figure 2), even for the vertical velocity at the surface for which the Stokesian approximation (B2) is totally meaningless. The Lagrangian approximation is also somewhat more accurate than its Eulerian counterpart, (B1). For moderately steep waves, the accuracy of the $N$ th-order Lagrangian approximation of the surface matches that of the Eulerian approximation of order $N+2$ (see also notes (v) and (vi) in Appendix A). 
(a)

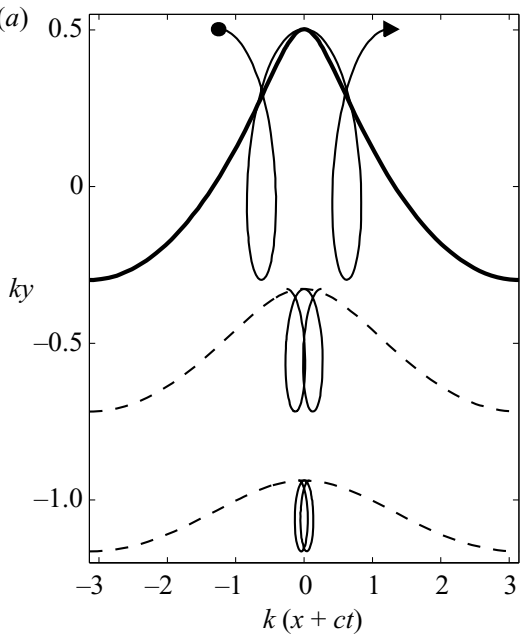

(b)

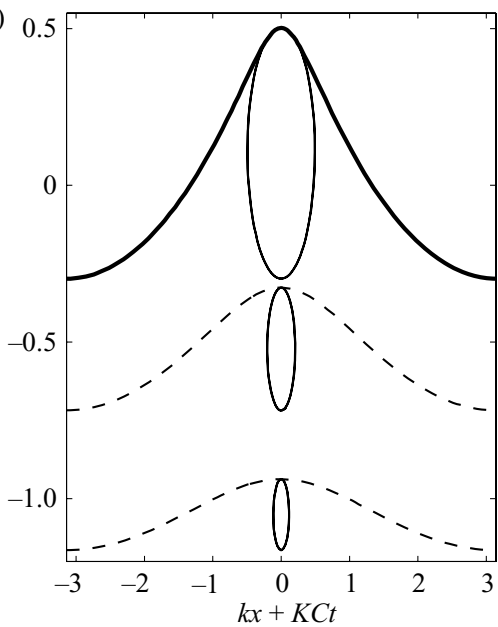

FiguRE 1. Streamlines and trajectories over two periods in deep water $(\omega=0, \varepsilon=0.4)$ : streamlines (iso- $\beta$ for $k \beta=-1 / 2,-1)$; - trajectories; - , free surface $(\beta=0)$. (a) Complete trajectories; $(b)$ trajectories minus mass transport.
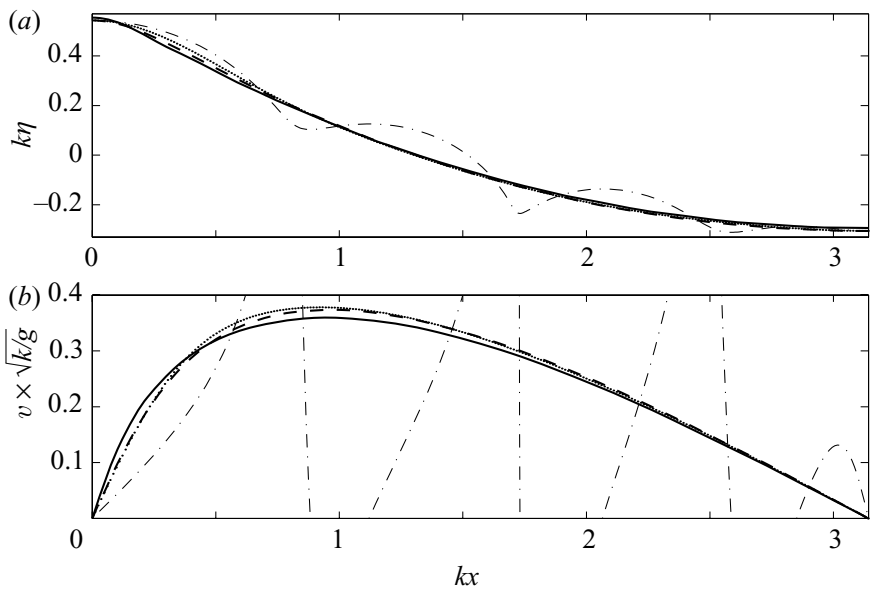

FIGURE 2. Deep-water seventh-order approximations and exact solution $(\omega=0, \varepsilon=0.424)$ : exact (Fenton 1988); ---, Lagrange (6.1)-(6.3); '- Euler (B 1); -- -, Stokes (B 2). (a) Surface elevation; $(b)$ vertical velocity at the surface.

The superiority of Stokes-like expansions in Eulerian variables over the Stokesian ones is clearly demonstrated by Drennan et al. (1992). The Lagrangian expansion is even better. The superiority of the Lagrangian formulation also appears in the Fourier coefficients of the surface elevation. To the leading order, the $n$th Fourier coefficients of $x$ and $\eta(6.3 a-b)$, i.e. respectively for $n \geqslant 2$

$$
\frac{(n+2) \varepsilon^{n+2}}{n(n+1) !}, \quad \frac{\varepsilon^{n+2}}{(n+1) !},
$$

are smaller and decay faster than their Eulerian and Stokesian counterparts (see Appendix B). This is an indication that the Lagrangian Stokes-like expansion may indeed have a larger radius of convergence than the Eulerian one and, a fortiori, 

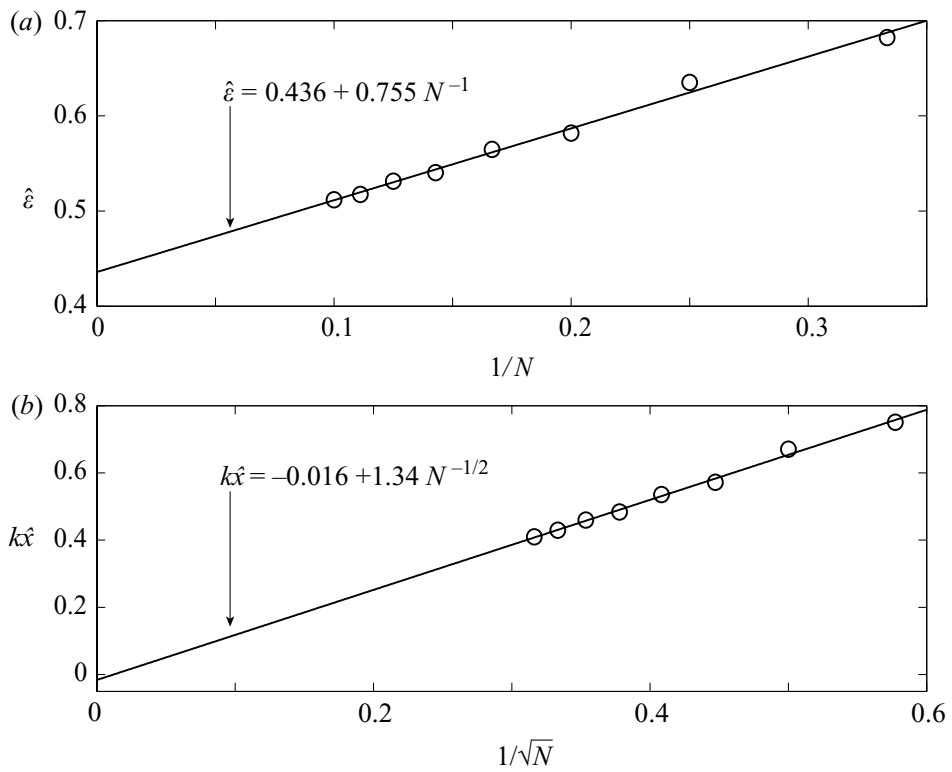

FiguRE 3. Convergence characteristics of the Lagrangian Stokes-like approximation of the highest irrotational wave in deep water: $\bigcirc$, third- to tenth-order approximations; - , linear regressions. (a) Steepness; (b) Absissa where $\eta_{x}=-1 / \sqrt{3}$.

of the Stokesian too. Thus, it would be interesting to determine whether or not the radius of convergence of the Lagrangian Stokes-like expansion includes the highest wave. Low-order approximations of the highest wave give some indications of this possibility.

\subsection{Highest wave}

The advantage of the Lagrangian Stokes-like approximation is more significant for the steepest waves, which cannot be computed with Fenton's program and which are poorly approximated by an Eulerian expansion of low order. For instance, the Eulerian approximations always have a smooth crest whereas the Lagrangian approximations predict a sharp crest for $\varepsilon=\hat{\varepsilon} \approx 1,0.5819,0.5119$ for the first-, fifth- and tenth-order approximations, respectively. The convergence of the $N$ th-order approximation $\hat{\varepsilon}_{N}$ (of the maximum steepness $\hat{\varepsilon}$ ) appears to be logarithmic with $\hat{\varepsilon}_{N} \sim \hat{\varepsilon}+\lambda / N$ as $N \rightarrow \infty$ where $\hat{\varepsilon} \approx 0.436$ and $\lambda \approx 0.755$ (figure $3 a$ ). Assuming that this is the case, a better estimation of the limit is obtained via the Richardson extrapolation $\hat{\varepsilon}_{N}^{\prime}=2 \hat{\varepsilon}_{2 N}-\hat{\varepsilon}_{N}$ giving $\hat{\varepsilon}_{5}^{\prime} \approx 0.44187$, which is less than $0.3 \%$ off the 'exact' one $\hat{\varepsilon} \approx 0.44316$ (Williams 1981).

All these approximations of the highest wave have a $0^{\circ}$ inner angle at the crest $-\mathrm{a}$ cusp such that $\eta(x)-\eta(0) \propto|x|^{2 / 3}$ locally - whereas the exact inner angle is $120^{\circ}$, i.e.

$$
\left.\frac{\mathrm{d} \eta}{\mathrm{d} x}\right|_{x=0^{ \pm}}=\frac{\mp 1}{\sqrt{3}} .
$$

Nevertheless, if $\hat{x}_{N}\left(0 \leqslant k \hat{x}_{N}<\pi\right)$ denotes the horizontal position where $\eta_{x}=-3^{-1 / 2}$ for the $N$ th-order Lagrangian approximation of the highest wave, the loworder approximations indicate the asymptotic behaviour $k \hat{x}_{N} \sim-0.016+1.34 N^{-1 / 2}$ (figure $3 b$ ) which is consistent with (6.7), i.e. it shows that probably $\hat{x}_{N} \rightarrow 0$ as $N \rightarrow \infty$, as it should be. 
Although further analyses are required, this brief investigation suggests that the Lagrangian Stokes-like expansion could be convergent for the highest wave. It also shows that the convergence should then be very slow (logarithmic). However, for practical calculations, the rate of convergence may be improved using another expansion parameter (Cokelet 1977) and via some efficient convergence improvement techniques (Weniger 1989).

\section{Application to irrotational waves on finite depth}

In finite depth, as for the case of deep water, we could use the Miche coordinates, i.e. coordinates such that $\beta=-\psi / c, C=c$ and $J=1$. However, with this particular choice, the algebra increases greatly with the order, and the third-order approximation is already too complicated to be reported here. An algebraically simpler solution is obtained with the alternative choice $K=k$ and $Y_{0}$ as a linear function of $\beta$, i.e. using the non-normalized simplified coordinates. To the third-order, we obtain

$$
\begin{aligned}
Y_{0}= & \beta+\frac{\beta+d}{2 k d \tanh k d} \varepsilon^{2}, \\
X_{1}= & \frac{\cosh k(\beta+d)}{k \sinh k d} \varepsilon+\frac{T(\beta+d) \sinh k(\beta+d)}{(1-S) \sinh k d} \varepsilon^{3} \\
& -\frac{\left(S^{3}+18 S+8\right) \cosh k(\beta+d)+6 S\left(4 S^{2}-3 S-1\right) \cosh 3 k(\beta+d)}{8 k(1-S)^{3} \sinh k d} \varepsilon^{3}, \\
Y_{1}= & \frac{\sinh k(\beta+d)}{k \sinh k d} \varepsilon+\frac{T(\beta+d) \cosh k(\beta+d)}{(1-S) \sinh k d} \varepsilon^{3} \\
& -\frac{-\left(5 S^{3}-8 S^{2}+22 S+8\right) \sinh k(\beta+d)+2 S\left(8 S^{2}-7 S-1\right) \sinh 3 k(\beta+d)}{8 k(1-S)^{3} \sinh k d} \varepsilon^{3}, \\
X_{2}= & \frac{S^{2}-S+3 S^{2} \cosh 2 k(\beta+d)}{2 k(1-S)^{2}} \varepsilon^{2}, \\
Y_{2}= & \frac{3 S^{2} \sinh 2 k(\beta+d)}{2 k(1-S)^{2}} \varepsilon^{2}, \\
X_{3}= & \frac{\left(34 S^{3}-38 S^{2}+4 S\right) \cosh k(\beta+d)+\left(33 S^{3}-6 S^{2}\right) \cosh 3 k(\beta+d)}{24 k(1-S)^{3} \sinh k d} \varepsilon^{3}, \\
Y_{3}= & \frac{6\left(S^{3}-S^{2}\right) \sinh k(\beta+d)+\left(11 S^{3}-2 S^{2}\right) \sinh 3 k(\beta+d)}{8 k(1-S)^{3} \sinh k d} \varepsilon^{3},
\end{aligned}
$$

and

$$
\begin{aligned}
\frac{C}{c_{0}} & =1+\frac{2+7 S^{2}-4 S(1-S) \cosh 2 k(\beta+d)}{4(1-S)^{2}} \varepsilon^{2}, \\
\frac{c}{c_{0}} & =1+\frac{2+7 S^{2}-4 T(1-S)}{4(1-S)^{2}} \varepsilon^{2}, \\
\frac{B}{c_{0}^{2}} & =1+\frac{2+2 S+5 S^{2}}{2(1-S)^{2}} \varepsilon^{2} \\
J & =1+\frac{T-S \cosh 2 k(\beta+d)}{1-S} \varepsilon^{2},
\end{aligned}
$$

where $c_{0}^{2} \equiv g k^{-1} \tanh k d, S \equiv \operatorname{sech} 2 k d$ and $T \equiv(2 k d)^{-1} \tanh 2 k d$. As for the deepwater case, this approximation is free of secular terms; it is hence uniformly valid for 


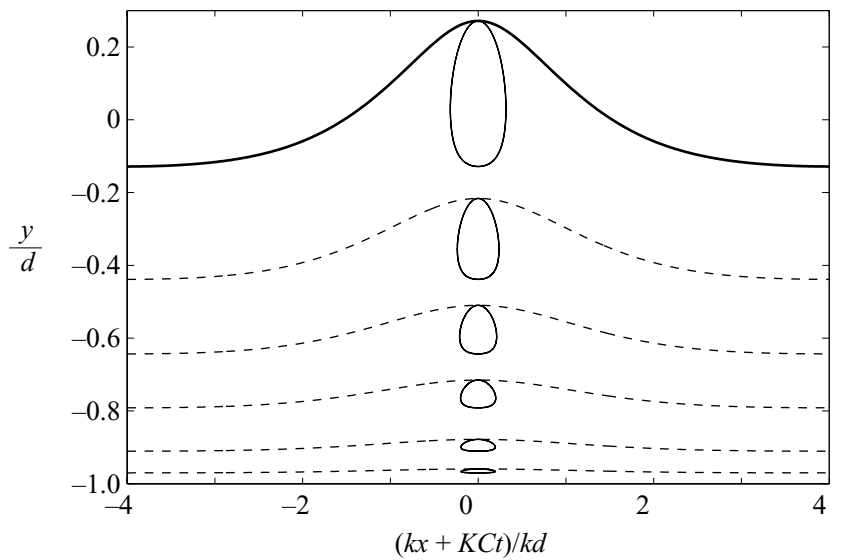

FIGURE 4. Streamlines and relative trajectories in finite depth $(\omega=0, \varepsilon=\pi / 20, k d=\pi / 4)$ : --- , streamlines; - , trajectories minus drift; - , free surface.

all times and this remains true at higher orders. At the free surface, we have

$$
\begin{aligned}
k x= & k \xi-\left[\frac{\varepsilon}{\tanh k d}+\frac{\left(8-54 S^{2}+19 S^{3}\right) \varepsilon^{3}}{8(1-S)^{3} \tanh k d}\right] \sin k \xi \\
& -\frac{\left(2 S+S^{2}\right) \varepsilon^{2}}{2(1-S)^{2}} \sin 2 k \xi+\frac{\left(8 S-34 S^{2}-S^{3}\right) \varepsilon^{3}}{24(1-S)^{3} \tanh k d} \sin 3 k \xi, \\
k \eta= & \frac{\varepsilon^{2}}{2 \tanh k d}+\left[\varepsilon+\frac{\left(4 S-14 S^{2}+17 S^{3}\right) \varepsilon^{3}}{8(1-S)^{3}}\right] \cos k \xi \\
& +\frac{3 k d S T \varepsilon^{2}}{(1-S)^{2}} \cos 2 k \xi-\frac{\left(4 S-14 S^{2}-17 S^{3}\right) \varepsilon^{3}}{8(1-S)^{8}} \cos 3 k \xi,
\end{aligned}
$$

and at the bottom

$$
C(-d)=c_{0}\left[1+\frac{2-4 S+11 S^{2}}{4(1-S)^{2}} \varepsilon^{2}\right]+O\left(\varepsilon^{4}\right),
$$

$C(-d)$ being the wave phase velocity observed in the frame of reference without net mass transport at the bottom. In this frame of reference, the drift velocity is

$$
\frac{V}{c_{0}}=\left[\frac{\varepsilon \sinh k(\beta+d)}{\sinh k d}\right]^{2}+O\left(\varepsilon^{4}\right) .
$$

Subtracting the mass transport, the (then closed) particle trajectories become more asymmetric (epitrochoidal-like, see figure 4) as the depth decreases and as the steepness increases (Wehausen \& Laitone 1960, §27 $\alpha$ ).

The third-order Lagrangian approximation is more accurate than the third-order Eulerian one, as demonstrated for the relatively steep and long wave example displayed in figure 5. For this particular example, the third-order Lagrangian surface is also more accurate than the fifth-order Eulerian approximation of Fenton (1990). This example demonstrates that Lagrangian approximations are accurate for a broader range of steepness and wavelength-to-depth ratio than their Eulerian counterparts. The differences are more pronounced for the steeper waves which cannot be computed with Fenton's (1988) program. Other evidence of the superiority of the Lagrangian approximations is given by Chang et al. (2007) (note that their thirdorder approximation is slightly different from the one presented here). 

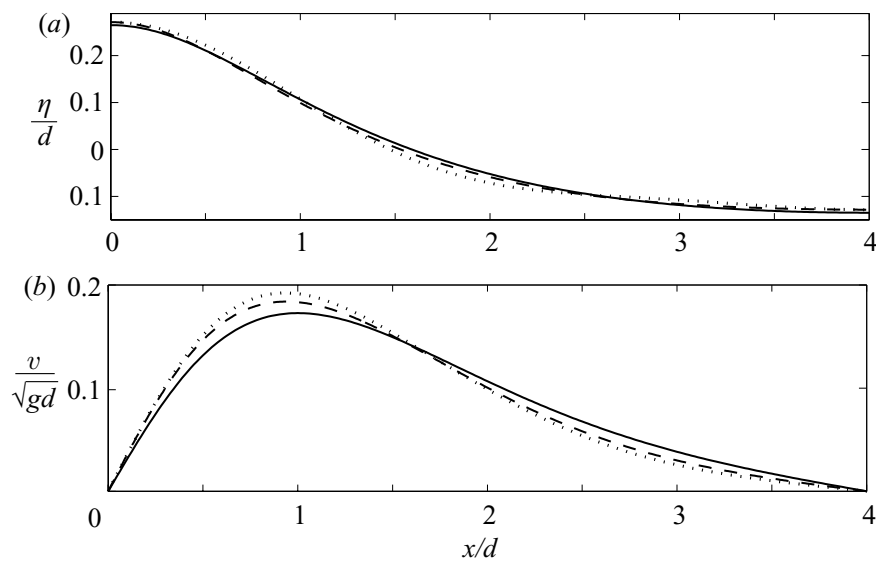

FIGURE 5. Finite-depth third-order approximations and exact solution $(\omega=0, \varepsilon=\pi / 20, k d=$ $\pi / 4)$ : - , exact (Fenton 1988); ---, Lagrange (7.1); - , Euler (Fenton 1985). (a) Surface elevation; $(b)$ vertical velocity at the surface.

\section{Application to Gerstner-like waves on finite depth}

An irrotational wave (with a non-zero amplitude) necessarily yields a net mass transport (Levi-Civita 1912; Constantin 2006). In deep water, Gerstner's (1802) exact solution is a rotational wave without Stokes' drift. The existence of a (rotational) wave with closed particle trajectories on finite depth was proved by Dubreil-Jacotin (1934). She showed that this solution is unique when the period is fixed, but the solution can be obtained explicitly only for deep water (Gerstner's wave). A thirdorder approximation was derived by Kravtchenko \& Daubert (1957).

Since there is no net mass transport, both $C$ and $K$ must be constant in the series (5.1). For the sake of simplicity, it is natural to take $K=k$ for a $(2 \pi / k)$-periodic wave (this is always possible via a change of definition of the $\alpha$-variable) and hence $C=c$. Kravtchenko \& Daubert (1957) derived their approximation under the constraint $J=1$ but, in order to compare with the irrotational approximation given in $\S 7$, it is better to take $Y_{0}$ as a linear function of $\beta$. Thus, to the third-order approximation we have

$$
\begin{aligned}
Y_{0}= & \beta+\frac{\beta+d}{2 k d \tanh k d} \varepsilon^{2} \\
X_{1}= & \frac{\cosh k(\beta+d)}{k \sinh k d} \varepsilon+\frac{\left(16(k d)^{2}\left(S-S^{2}\right) T-4-32 S+16 S^{2}-7 S^{3}\right) \cosh k(\beta+d)}{8 k(1-S)^{3} \sinh k d} \varepsilon^{3} \\
& +\frac{\left(1-S^{2}-4(k d)^{2} S T\right)(\beta+d) \sinh k(\beta+d)}{4(k d)^{2} T(1-S) \sinh k d} \varepsilon^{3}+\frac{15 S^{2} \cosh 3 k(\beta+d)}{4 k(1-S)^{2} \sinh k d} \varepsilon^{3} \\
Y_{1}= & \frac{\sinh k(\beta+d)}{k \sinh k d} \varepsilon+\frac{\left(16(k d)^{2}\left(S-S^{2}\right) T-4-28 S+8 S^{2}-3 S^{3}\right) \sinh k(\beta+d)}{8 k(1-S)^{3} \sinh k d} \varepsilon^{3} \\
& +\frac{\left(1-S^{2}-4(k d)^{2} S T\right)(\beta+d) \cosh k(\beta+d)}{4(k d)^{2} T(1-S) \sinh k d} \varepsilon^{3}+\frac{9 S^{2} \sinh 3 k(\beta+d)}{4 k(1-S)^{2} \sinh k d} \varepsilon^{3} \\
X_{2}= & \frac{S^{2}-S+3 S^{2} \cosh 2 k(\beta+d)}{2 k(1-S)^{2}} \varepsilon^{2} \\
Y_{2}= & \frac{3 S^{2} \sinh 2 k(\beta+d)}{2 k(1-S)^{2}} \varepsilon^{2}
\end{aligned}
$$




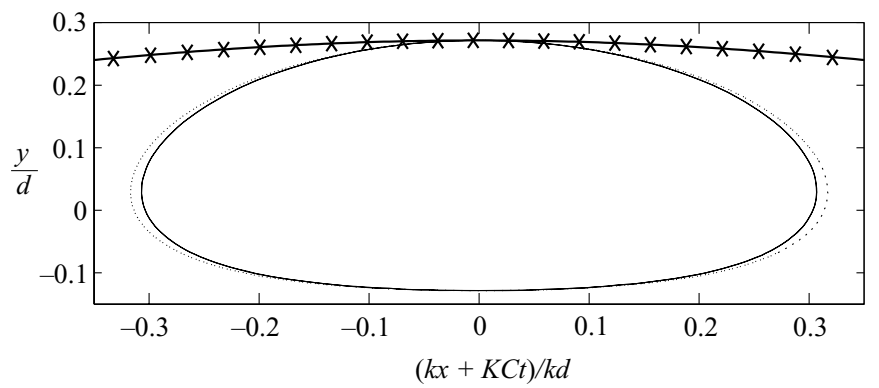

FiguRE 6. Comparison between finite-depth third-order Gerstner and irrotational approximations $(\varepsilon=\pi / 20, k d=\pi / 4):-$, Gerstner surface; $\times$, irrotational surface; 一, Gerstner trajectory; $\cdots$, irrotational trajectories after substraction of the Stokes drift.

$$
\begin{aligned}
& X_{3}=\frac{\left(34 S^{3}-38 S^{2}+4 S\right) \cosh k(\beta+d)+\left(33 S^{3}-6 S^{2}\right) \cosh 3 k(\beta+d)}{24 k(1-S)^{3} \sinh k d} \varepsilon^{3}, \\
& Y_{3}=\frac{6\left(S^{3}-S^{2}\right) \sinh k(\beta+d)+\left(11 S^{3}-2 S^{2}\right) \sinh 3 k(\beta+d)}{8 k(1-S)^{3} \sinh k d} \varepsilon^{3},
\end{aligned}
$$

and

$$
\begin{aligned}
\frac{c}{c_{0}} & =1+\frac{S\left(2+9 S+7 S^{2}-8(k d)^{2} S T\right)}{4(1+S)(1-S)^{2}} \varepsilon^{2}, \\
\frac{B}{c_{0}^{2}} & =1+\frac{4+4 S+5 S^{2}+5 S^{3}-8(k d)^{2} S^{2} T}{2(1+S)(1-S)^{2}} \varepsilon^{2}, \\
J & =1+\left[\frac{T}{1+S}-\frac{S \cosh 2 k(\beta+d)}{1-S}\right] \varepsilon^{2}, \\
\frac{\omega}{k c_{0}} & =\frac{\sinh 2 k(\beta+d)}{\sinh ^{2} k d} \varepsilon^{2},
\end{aligned}
$$

where $c_{0}^{2} \equiv g k^{-1} \tanh k d, S \equiv \operatorname{sech} 2 k d$ and $T \equiv(2 k d)^{-1} \tanh 2 k d$. Note that the phase speed varies with the amplitude, except in the deep-water limit where the Gerstner wave is recovered.

This approximation is similar to the third-order irrotational one and is also free of secular terms. They are very close (figure 6) except, perhaps, in shallow water $(k d \ll 1)$ for which the Stokes-like expansions are inefficient.

\section{Discussion}

For the classical Stokes Lagrangian second-order approximation, it was shown that steadiness is approximately (but not identically) fulfilled. This raises the question of the definition of a steady flow in the Lagrangian description of motion. The exact general mathematical form of a steady flow was then derived for an incompressible fluid in two-dimensional motion. Some simplified expressions, more convenient for most practical applications, were also derived. It was thus shown that considering the wave velocity as a function of the 'vertical' label $\beta$ is a possible consistent choice for describing mass transport when both $\psi$ and $J$ depend on $\beta$ only, but it is not the only possibility. 
Once the Lagrangian form of a steady motion has been clearly defined, it is easy to introduce a Lagrangian extension of the Stokes expansion. This perturbation scheme allowed the derivation of approximations that are uniformly valid for all times. This was demonstrated, for irrotational waves, with a seventh-order approximation for deep water and a third-order approximation for finite depth and, for rotational waves, with a third-order approximation with closed particle orbits for finite depth (Gerstner-like wave). It was further shown that Lagrangian approximations are more accurate than the Eulerian and Stokesian approximations of the same order. At least for irrotational waves in deep water, the low-order approximations suggest that the Lagrangian Stokes-like expansion could possibly be convergent for the highest wave.

The Lagrangian Stokes-like expansion has been illustrated with various choices. In deep water, the Miche variables were used, i.e. we took $C=c$ and $J=1$. On finite depth, we took $K=k$ and $J \neq 1$ because it yields algebraically simpler approximations. Though the latter choice may seem more intuitive, it is not necessarily the most convenient, however. Indeed, to obtain the value of $\beta$ corresponding to a given streamline $\psi=\mathrm{Cst}$, for example, one must solve the equation $\psi=-\int J(\beta) C(\beta) \mathrm{d} \beta$, which is not convenient for practical applications. Conversely, with the Miche variables, the $\beta$-label is explicitly given by $\beta=-\psi / c$. Moreover, the solution being a function of $K \alpha-\Omega t$ (with $\Omega \equiv K C$ ), the $\alpha$ and $t$ variables play a symmetrical role and it is therefore neither more 'logical', nor 'natural', to take $K=k$ instead of $C=c$; the most convenient choice depends on the problem at hand.

The present study is limited to two-dimensional motions. Although the simplified coordinates of $\S 4.3$ can be used for three-dimensional surface waves (Abrashkin 1996), the general mathematical Lagrangian form of steady motions in three dimensions may be of some practical interest. This generalization is given in Appendix C, together with the extension for compressible fluids.

We have seen that Lagrangian perturbation techniques can be used to derive approximations that are uniformly valid for long times. This is certainly also true for unsteady motions, provided that the approximation correctly represents the phenomenon under investigation. It was shown here that defining a steady flow is not as trivial as it may sound at first. The Lagrangian definition of a given unsteady flow will be a more complicated task, in general. Actually, there are few unsteady flows (e.g. standing waves) that have a precise definition. For such well-defined flows, we should be able to derive their correct Lagrangian mathematical definition. More often, complex unsteady flows are not so well-defined, however, and their analysis is a matter of interpretation and viewpoint, which are not unique. For instance, freak waves (Kharif \& Pelinovsky 2003) are an intensively studied phenomenon, but there is no (mathematically) precise definition of a freak wave.

The author is grateful to Christopher J. Lawrence and to the referees for their comments.

\section{Appendix A. Remarks on Gerstner's waves}

The Gerstner (1802) wave is a well-known exact solution for rotational waves in deep water. In the frame of reference where the flow is steady, the $(2 \pi / k)$-periodic Gerstner wave is

$$
\begin{array}{r}
k x=k \xi-\varepsilon \mathrm{e}^{k \beta} \sin k \xi, \quad k y=k \beta+\frac{1}{2} \varepsilon^{2}+\varepsilon \mathrm{e}^{k \beta} \cos k \xi, \\
J=1-\varepsilon^{2} \mathrm{e}^{2 k \beta}, \quad \omega=2 k c \varepsilon^{2} \mathrm{e}^{2 k \beta}\left(1-\varepsilon^{2} \mathrm{e}^{2 k \beta}\right)^{-1},
\end{array}
$$


where $\xi=\alpha-c t, c^{2}=g / k$ and $\varepsilon$ is half the total wave height multiplied by $k$. Note that:

(i) The term $\varepsilon^{2} / 2$ in $(\mathrm{A} 1 b)$ is generally omitted in the literature, but is introduced here to ensure that $\eta=y(\xi, \beta=0)$ averages zero according to (2.1).

(ii) The particle horizontal position $x_{F}$ observed in the frame of reference where there is no mass transport as $\beta \rightarrow-\infty$ (where the wave appears to travel with speed $c)$ is $x_{F}=x+c t=\alpha-k^{-1} \varepsilon \mathrm{e}^{k \beta} \sin k(\alpha-c t)$, so the particle trajectories are closed and there is no net mass transport (in this frame of reference).

(iii) Gerstner's solution can be rewritten such that $J=1$ via a change of coordinate $(\alpha, \beta) \mapsto\left(\alpha, \beta^{\prime}\right)$ with

$$
\beta^{\prime}=\beta+\frac{\varepsilon^{2}}{2 k}\left[1-\mathrm{e}^{2 k \beta}\right] \Longleftrightarrow \beta=\beta^{\prime}-\frac{\varepsilon^{2}}{2 k}-\frac{1}{2 k} \mathrm{~W}\left(-\varepsilon^{2} \mathrm{e}^{2 k \beta^{\prime}-\varepsilon^{2}}\right),
$$

where $\mathrm{W}$ is the Lambert function (Corless et al. 1996). Thus, Gerstner's solution becomes

$$
\begin{aligned}
& k x=k \xi-\left[-\mathrm{W}\left(-\varepsilon^{2} \mathrm{e}^{2 k \beta^{\prime}-\varepsilon^{2}}\right)\right]^{1 / 2} \sin k \xi, \\
& k y=k \beta^{\prime}-\frac{1}{2} \mathrm{~W}\left(-\varepsilon^{2} \mathrm{e}^{2 k \beta^{\prime}-\varepsilon^{2}}\right)+\left[-\mathrm{W}\left(-\varepsilon^{2} \mathrm{e}^{2 k \beta^{\prime}-\varepsilon^{2}}\right)\right]^{1 / 2} \cos k \xi,
\end{aligned}
$$

which is substantially more complicated than (A 1).

(iv) The Gerstner wave has isobaric streamlines, i.e. $P=P(\psi)$. Dubreil-Jacotin (1932) proved that there is no such solution on finite depth. For a homogeneous adiabatic compressible fluid, Kiebel (1933) proved that the Gerstner wave is the only wave with isobaric streamlines. Dubreil-Jacotin (1935) extended Kiebel's result to heterogeneous incompressible fluids. Gouyon (1958) gave a simple proof for homogeneous fluids, and Kalisch (2004) rediscovered this result.

(v) Gerstner's wave is also the linear approximation of an irrotational wave. Eliminating $\xi$ between $x$ and $y$ at the surface $\beta=0$ and expanding in power series of $\varepsilon$ up to the fourth order, we obtain

$$
k \eta=\varepsilon \cos k x+\frac{1}{2} \varepsilon^{2} \cos 2 k x+\frac{3}{8} \varepsilon^{3}(\cos 3 k x-\cos k x)+\frac{1}{3} \varepsilon^{4}(\cos 4 k x-\cos 2 k x) .
$$

The only difference with the fourth-order Eulerian approximation, (B 1b), lies in the sign of the term $\varepsilon^{4} \cos 2 k x / 3$. Hence, the surface first-order Lagrangian approximation includes the third-order Eulerian approximation.

(vi) Further, the expansion (A 4) is of the form

$$
k \eta=\sum_{n=1}^{\infty} \frac{n^{n-1}}{n ! 2^{n-1}}\left[\varepsilon^{n}+O\left(\varepsilon^{n+2}\right)\right] \cos n k x,
$$

which, to the leading order, is identical to the irrotational Stokes expansion in deep water (see Appendix B). Hence, the Gerstner wave captures some higher-order features of the Stokes wave.

(vii) The approximately trochoidal profile of the Stokes wave in deep water has long been noted (Stokes 1847; Lamb 1932, art. 250). Constantin (2001) observes that the Gerstner wave presents features of general interest for deep water waves, and Leblanc (2004) investigated its local stability.

\section{Appendix B. Eulerian and Stokesian seventh-order approximations}

Introducing the complex potential $f=\phi+\mathrm{i} \psi$ and the complex variable $z=x+\mathrm{i} y$, the seventh-order Stokes-like approximation for an irrotational wave in deep water 
and in the Eulerian description of motion (called here the Eulerian approximation) is

$$
\begin{aligned}
f= & -c z+\mathrm{i} \sqrt{g / k^{3}}\left[-\frac{1}{2} \varepsilon^{2}+\frac{1}{4} \varepsilon^{4}+\frac{35}{48} \varepsilon^{6}+\left(\varepsilon-\frac{1}{2} \varepsilon^{3}-\frac{37}{24} \varepsilon^{5}-\frac{4267}{1440} \varepsilon^{7}\right) \mathrm{e}^{-\mathrm{i} k z}\right. \\
& +\left(\frac{1}{2} \varepsilon^{4}+\frac{1}{6} \varepsilon^{6}\right) \mathrm{e}^{-2 \mathrm{i} k z}+\left(\frac{1}{12} \varepsilon^{5}+\frac{73}{144} \varepsilon^{7}\right) \mathrm{e}^{-3 \mathrm{i} k z} \\
& \left.+\frac{1}{72} \varepsilon^{6} \mathrm{e}^{-4 \mathrm{i} k z}+\frac{1}{480} \varepsilon^{7} \mathrm{e}^{-5 \mathrm{i} k z}\right], \\
k \eta= & \left(\varepsilon-\frac{3}{8} \varepsilon^{3}-\frac{211}{192} \varepsilon^{5}-\frac{14411}{5120} \varepsilon^{7}\right) \cos k x+\left(\frac{1}{2} \varepsilon^{2}+\frac{1}{3} \varepsilon^{4}-\frac{13}{48} \varepsilon^{6}\right) \cos 2 k x \\
& +\left(\frac{3}{8} \varepsilon^{3}+\frac{99}{128} \varepsilon^{5}+\frac{3783}{5120} \varepsilon^{7}\right) \cos 3 k x+\left(\frac{1}{3} \varepsilon^{4}+\frac{217}{180} \varepsilon^{6}\right) \cos 4 k x \\
& +\left(\frac{125}{384} \varepsilon^{5}+\frac{15769}{9216} \varepsilon^{7}\right) \cos 5 k x+\frac{27}{80} \varepsilon^{6} \cos 6 k x+\frac{16807}{46080} \varepsilon^{7} \cos 7 k x, \\
c= & \sqrt{g / k}\left[1+\frac{1}{2} \varepsilon^{2}+\frac{1}{8} \varepsilon^{4}+\frac{1}{16} \varepsilon^{6}\right] .
\end{aligned}
$$

Note that, after elimination of $K \xi$ and expansion up to the seventh order, the equations $(6.3)$ yield (B $1 b)$.

Stokes (1880) also derived a fifth-order approximation using $\phi$ and $\psi$ as independent variables. Introducing the more convenient complex variable $\zeta=-f / c$, the seventhorder Stokes' expansion (called here the Stokesian approximation) is

$$
\begin{aligned}
k z= & k \zeta-\frac{\mathrm{i}}{2} \varepsilon^{2}+\frac{\mathrm{i}}{2} \varepsilon^{4}+\frac{13 \mathrm{i}}{24} \varepsilon^{6}+\mathrm{i}\left(\varepsilon-\frac{3}{2} \varepsilon^{3}-\frac{1}{24} \varepsilon^{5}-\frac{3007}{1440} \varepsilon^{7}\right) \mathrm{e}^{-\mathrm{i} k \zeta} \\
& +\mathrm{i}\left(\varepsilon^{2}-\frac{5}{2} \varepsilon^{4}+\frac{19}{12} \varepsilon^{6}\right) \mathrm{e}^{-2 \mathrm{i} k \zeta}+\mathrm{i}\left(\frac{3}{2} \varepsilon^{3}-\frac{31}{6} \varepsilon^{5}+\frac{113}{18} \varepsilon^{7}\right) \mathrm{e}^{-3 \mathrm{i} k \zeta} \\
& +\mathrm{i}\left(\frac{8}{3} \varepsilon^{4}-\frac{839}{72} \varepsilon^{6}\right) \mathrm{e}^{-4 \mathrm{i} k \zeta}+\mathrm{i}\left(\frac{125}{24} \varepsilon^{5}-\frac{39647}{1440} \varepsilon^{7}\right) \mathrm{e}^{-5 \mathrm{i} k \zeta} \\
& +\frac{54 \mathrm{i}}{5} \varepsilon^{6} \mathrm{e}^{-6 \mathrm{i} k \zeta}+\frac{16807 \mathrm{i}}{720} \varepsilon^{7} \mathrm{e}^{-7 \mathrm{i} k \zeta} .
\end{aligned}
$$

All the approximations above use the steepness $\varepsilon$ as the expansion parameter, but other expansion parameters can also be used. For instance, Stokes used the coefficient of the fundamental frequency of $k \eta$ in his expansion in the $(x, y)$-plane (Stokes 1847, $\S 13)$ and the coefficient of the fundamental frequency of $k z$ in his expansion in the $(\phi, \psi)$-plane (Stokes 1880, equations 17-18). However, Schwartz (1974) has found that Stokes' expansion parameters do not increase monotonically with the wave height and, therefore, using the steepness is more convenient. Other expansion parameters are more efficient in terms of radius and rate of convergence (see e.g. Cokelet 1997), but they are less practical than the steepness. Indeed, the steepness is easily obtained from experimental data by direct measurement of the wavelength and height, whereas the determination of other parameters may require more measurements (e.g. the velocity field) and more mathematical treatment (e.g. Fourier transform).

Wilton (1914) noted that, to the leading order, the $n$th Fourier coefficient in the expansion (B 2) is $\varepsilon^{n} n^{n-1} / n$ ! for all $n \geqslant 1$ (up to infinity). This should be compared with its Eulerian counterpart (B 1) - where the corresponding coefficient is $\varepsilon^{n} n^{n-1} / n ! 2^{n-1}$ $(\forall n \geqslant 1)$ for the surface (B $1 b)$ and $\varepsilon^{n+2} / n !(n-1)(\forall n \geqslant 2)$ for the potential (B $\left.1 a\right)-$ which has faster decaying Fourier coefficients than the Stokesian one, at least to the leading order. It is shown in $\S 6$ that the Lagrangian Stokes-like expansion of the free surface has even more rapidly decaying leading-order terms.

\section{Appendix C. Steady three-dimensional flows}

The derivations of $\S 4.1$ are briefly generalized here for three-dimensional flows and subsequently for compressible fluids.

In the Eulerian description, let $\boldsymbol{x}=\left(x_{1}, x_{2}, x_{3}\right)$ be the Cartesian coordinate and let $\boldsymbol{u}(\boldsymbol{x}) \equiv \mathrm{D} \boldsymbol{x} / \mathrm{D} t$ be the velocity field. The fluid incompressibility $(\nabla \cdot \boldsymbol{u}=0)$ is fulfilled introducing two (scalar) streamfunctions $\psi(\boldsymbol{x})$ and $\lambda(\boldsymbol{x})$ such that $\boldsymbol{u}=(\nabla \psi) \wedge(\nabla \lambda)$ 
(Aris 1962). It can be seen easily that $\mathrm{D} \psi / \mathrm{D} t=\mathrm{D} \lambda / \mathrm{D} t=0$, hence the streamfunctions do not depend on time following the motion.

In Lagrangian description, let $\boldsymbol{\alpha}=\left(\alpha_{1}, \alpha_{2}, \alpha_{3}\right)$ be the particle labels, $J(\boldsymbol{\alpha})$ being the Jacobian of the transformation $\boldsymbol{x}(t=0) \mapsto \boldsymbol{\alpha}$. The definition of the streamfunctions and the incompressibility yield

$$
u_{i}=\frac{\partial\left(x_{i}, \psi, \lambda\right)}{\partial\left(x_{1}, x_{2}, x_{3}\right)} \Longleftrightarrow \frac{\partial x_{i}}{\partial t}=\frac{1}{J} \frac{\partial\left(x_{i}, \psi, \lambda\right)}{\partial\left(\alpha_{1}, \alpha_{2}, \alpha_{3}\right)} .
$$

Because $J, \psi$ and $\lambda$ do not depend on $t,(\mathrm{C} 1 b)$ can be easily solved taking, for example, $\left(\alpha_{1}, \psi, \lambda, t\right)$ as independent variables. Thus, returning to the independent variables $(\boldsymbol{\alpha}, t)$ after integration, the general solution of $(\mathrm{C} 1 b)$ is

$$
\boldsymbol{x}=\boldsymbol{x}(\tau, \psi, \lambda), \quad \tau \equiv t+T(\boldsymbol{\alpha}), \quad \frac{\partial(T, \psi, \lambda)}{\partial\left(\alpha_{1}, \alpha_{2}, \alpha_{3}\right)}=J . \quad(\mathrm{C} 2 a-c)
$$

(C 2) is the general Lagrangian form of a three-dimensional steady flow for an incompressible fluid. Special forms of $T(\alpha)$ can be obtained relabelling the particles as in the two-dimensional case (see $\S 4)$.

The generalization for a compressible fluid of density $\rho$ is straightforward because, the mass conservation yielding $\nabla \cdot(\rho \boldsymbol{u})=0$, it is sufficient to introduce two streamfunctions $\{\Psi ; \Lambda\}$ such that $\rho \boldsymbol{u}=(\nabla \Psi) \wedge(\nabla \Lambda)$ and to proceed as above. Doing so, we obtain

$$
\boldsymbol{x}=\boldsymbol{x}(\tau, \Psi, \Lambda), \quad \tau \equiv t+T(\boldsymbol{\alpha}), \quad \frac{\partial(T, \Psi, \Lambda)}{\partial\left(\alpha_{1}, \alpha_{2}, \alpha_{3}\right)}=J \rho_{0},
$$

where $\rho_{0}(\boldsymbol{\alpha}) \equiv \rho(\boldsymbol{\alpha}, t=0)$.

\section{REFERENCES}

Abrashkin, A. A. 1996 Three-dimensional Gouyon waves. Fluid Dyn. 31, 583-587.

Abrashinin, A. A. \& Zen'Kovich, D. A. 1990 Vortex stationary waves on a shear flow. Izv. Akad. Nauk SSSR, Fiz. Aanos. Okeana 26, 35-46. (In Russian.)

ArIs, R. 1962 Vectors, Tensors, and the Basic Equations of Fluid Mechanics. Dover.

Buldakov, E. V., TAYloR, P. H. \& EATOCK-TAYlOR, R. 2006 New asymptotic description of nonlinear water waves in Lagrangian coordinates. J. Fluid Mech. 562, 431-444.

Chang, H. K., Liou, J. C. \& Su, M. Y. 2007 Particle trajectory and mass transport of finite-amplitude waves in water of uniform depth. Eur. J. Mech. B/Fluids 26, 385-403.

Cokelet, E. D. 1977 Steep gravity waves in water of arbitrary uniform depth. Phil. Trans. R. Soc. Lond. 286, 183-230.

Constantin, A. 2001 On deep water wave motion. J. Phys. A: 34, 1405-1417.

Constantin, A. 2006 The trajectories of particles in Stokes waves. Invent. Math. 166, 523-535.

Corless, R. M., Gonnet, G. H., Hare, D. E. G., Jefreey, D. J. \& Knuth, D. E. 1996 On the Lambert W function. Adv. Comput. Math. 5, 329-359.

Craik, A. D. D. 2005 George Gabriel Stokes on water wave theory. Annu. Rev. Fluid Mech. 37, 23-42.

DiAs, F. \& BRIDGES, T. J. 2006 The numerical computation of freely propagating time-dependent irrotational water waves. Fluid Dyn. Res. 38, 803-830.

Drennan, W. M., Hui, W. H. \& Tenti, G. 1992 Accurate calculations of Stokes water waves of large amplitude. Z. Angew. Math. Phys. 43, 367-384.

Dubreil-Jacotin, M.-L. 1932 Sur les ondes de type permanent dans les liquides hétérogènes. Accad. Naz. Lincei (6) 15, 814-819. (In French.)

Dubreil-Jacotin, M.-L. 1934 Sur la détermination rigoureuse des ondes permanentes périodiques d'ampleur finie. J. Math. 13, 217-291. (In French.) 
Dubreil-JaCotin, M.-L. 1935 Complément à une note antérieure sur les ondes de type permanent dans les liquides hétérogènes. Rend. Accad. Naz. Lincei (6) 21, 344-346. (In French.)

Fenton, J. D. 1988 The numerical solution of steady water wave problems. Comput. Geosci. 143, 357-368.

Fenton, J. D. 1990 Nonlinear wave theories. The Sea vol. 9, pp. 3-25. Wiley.

Fenton, J. D. 1999 Numerical methods for nonlinear waves. Adv. Coastal Ocean Engng 5, 241-324. World Scientific.

Gerstner, F. 1802 Theorie der Wellen. Abhand. Kön. Böhmischen Gesel. Wiss. Prague (in German).

Gouyon, R. 1958 Contribution à la théorie des houles. Thèse, Université de Toulouse, France (in French).

Kalisch, H. 2004 Periodic traveling water waves with isobaric streamlines. J. Nonlinear Math. Phys. 11, 461-471.

Kharif, C. \& Pelinovsky, E. 2003 Physical mechanisms of the rogue wave phenomenon. Eur. J. Mech. B/Fluids 22, 603-634.

Kiebel, I. A. 1933 On some two-dimensional motions of a heavy compressible fluid. Prikl. Math. Mech. 1, 51-55. (In Russian.)

Kravtchenko, J. \& Daubert, A. 1957 Closed trajectory waves in a finite depth. La Houille Blanche 3, 408-429. (In French.)

Lamb, H. 1932 Hydrodynamics. Cambridge University Press.

Leblanc, S. 2004 Local stability of Gerstner's waves. J. Fluid Mech. 506, 245-254.

Levi-Civita, T. 1912 Sulle onde di canale. Rend. Accad. Naz. Lincei (5) 21, 3-14. (In Italian.)

Longuet-Higgins, M. S. 1986 Eulerian and Lagrangian aspects of surface waves. J. Fluid Mech. 173, 683-707.

Longuet-Higgins, M. S. 1987 Lagrangian moments and mass transport in Stokes waves. J. Fluid Mech. 179, 547-555.

Miche, M. 1944 Mouvements ondulatoires de la mer en profondeur constante ou décroissante. Ann. Ponts Chaussées 114, 25-78, 131-164, 270-292, 369-406. (English translation: Univ. Calif. Wave Res. Lab. 3, 363, 1954.)

SCHWARTZ, L. W. 1974 Computer extended and analytic continuation of Stokes' expansion for gravity waves. J. Fluid Mech. 62, 553-578.

Stokes, G. G. 1847 On the theory of oscillatory waves. Trans. Camb. Phil. Soc. 8, 441-455.

Stokes, G. G. 1880 Supplement to a paper on the theory of oscillatory waves. G.G. Stokes Math. \& Phys. Papers, vol. 1, pp. 314-326. Cambridge University Press.

Wehausen, J. V. \& Laitone, E. V. 1960 Surface waves. Handbuch Phys. 9, 446-778. Springer.

WENIGER, E. J. 1989 Nonlinear sequence transformations for the acceleration of convergence and the summation of divergent deries. Comput. Phys. Rep. 10, 189-371.

Williams, J. M. 1981 Limiting gravity waves in water of finite depth. Phil. Trans. R. Soc. Lond A 302 (1466), 139-188.

Wilton, J. R. 1914 On deep water waves. Phil. Mag. S. VI 27 (158), 385-394. 